\title{
Erratum to: Discrete and Computational Geometry and Graphs
}

\author{
Jin Akiyama ${ }^{1(\bowtie)}$, Hiro Ito $^{2}$, Toshinori Sakai ${ }^{3}$, and Yushi Uno ${ }^{4}$ \\ 1 Tokyo University of Science, Tokyo, Japan \\ ja@jin-akiyama.com \\ 2 The University of Electro-Communications, Tokyo, Japan \\ ${ }^{3}$ Tokai University, Tokyo, Japan \\ ${ }^{4}$ Osaka Prefecture University, Sakai, Japan
}

\section{Erratum to:}

J. Akiyama et al. (Eds.):

Discrete and Computational Geometry and Graphs, LNCS, DOI: $10.1007 / 978-3-319-48532-4$

The sequence of the editor names was incorrect. Yushi Uno was not listed as volume editor. The updated online version of the cover and the front matter pages III-V can be found at DOI: 10.1007/978-3-319-48532-4 